\title{
AN ACTIVE VISION SYSTEM FOR MODELLING BIOMATERIALS
}

\author{
K. ALEMZADEH, L. ZHANG \& A. CONN \\ Department of Mechanical Engineering, University of Bristol, UK.
}

\section{ABSTRACT}

This paper describes an active vision system based reverse engineering approach to extract the 3D geometric information from dental cast/teeth and transfer this information into computer-aided design/computer-aided manufacture (CAM) systems to improve the accuracy of the constructed units for patient care. The benefits of our approach are a non-contact measurement, low cost, and capturing all the surface data at the same time. Our system involves the development of a vision rig prototype accommodating up to three charged couple device cameras, image processing, and shape recovery from a series of convex profile (2D) images of complex objects morphology (e.g. incisor or sunflower seed). The rig is designed using engineering design methods such as a concept selection matrix and a weighted objectives evaluation chart. The rig concept design is based on the human/stereo vision principles in computer vision. The occluding boundary technique and its derived method, the slicing method, are described and employed by the vision system to generate (3D) models of two typical teeth using a dental cast. Comparison of the vision system and the Renishaw Triclone dental system is demonstrated on these two models with one of them being manufactured using CAM technology. The results have shown that our vision system is capable of supporting the advanced reverse design applications in restorative dentistry. It is concluded that the new system could encode the shape of small complex objects and the device could be useful in the characterization of natural materials as a basis for biology-based design.

Keywords: advanced reverse design, modelling biomaterials, slicing method, vision system design.

\section{INTRODUCTION}

Reverse engineering (RE) is an advanced design technique that is used to create $3 \mathrm{D}$ free form surfaces from an existing physical model using a laser or contact probe as a surface digitizer [1]. RE is used as a powerful tool for initial conceptual and aesthetic design, product prototyping, performance testing, and model modification needed for obtaining an optimal product design [2]. However, traditional RE digitizing/scanning technologies such as the laser scanner and the coordinate measuring machine (CMM) both have their own limitations in surface digitizing [3]. Their data capturing process is slow, because the surface digitizing is done line-by-line and point-to-point. Our new optical/vision system has advantages in both digitizing mode (i.e. non-contact, fast, and capturing surface data) and application capability in bioengineering. The vision system uses an optical scanning method similar to a laser probe but it is safer for the user and the environment.

For decades, in dentistry, attempts have been made to use ceramics for fabrication of crowns and inlay material in restoration. However, it was only a few years ago that technologies that could be used successfully in dental practices became available. Two crucial obstacles made it intractable to use ceramics as an inlay/crown material:

- Appropriate adhesive technology to enable a lasting connection between the inlay or crown and the tooth.

- Capable systems that enable well-fitting inlays and crowns to be fabricated [4].

The second obstacle triggered a research trend towards the importance of 3D tooth modelling. It has been recognized that resistance to restoration failure is not solely a biological concern (e.g. toxicity), but that the cavity shape, dimensions, and the state of stress must all be taken into account [5-8].

The use of vision systems in dentistry predominantly centres on dental restoration [5]. This involves the fabrication of crowns and ceramic inlays through to complete mouth restructuring $[5,6]$. The vision 
system developed at the University of Bristol focuses on developing an off-line 3D accurate tooth modelling using a dental cast to fabricate crown/inlay in dental computer-aided design/computeraided manufacture (CAD/CAM). The aim is to extract the geometric information from teeth and transfer this information into CAD/CAM systems to improve the accuracy of the construction units in patient care, and at the same time to lower manufacturing costs by simplifying the method and reducing the production time. The experimental results are encouraging and support further development of image-based 3D depth recovery based on human vision and its applications in bioengineering modelling.

\subsection{The rig design}

\section{VISION RIG PROTOTYPE}

A vision rig was built according to the human/stereo vision principles (i.e. 3D depth recovery) $[9,10]$ to accommodate up to three charged couple device (CCD) cameras and a turntable (with the ability to turn $360^{\circ}$ ) for holding complex physical objects (e.g. incisor or sunflower seed) for image acquisition. The horizontal camera was used at this stage for the slicing method with a turntable to capture (2D) convex images. The two extra cameras created flexibility for further implementation of the stereo vision technique to deal with concave surfaces such as molars. The rig is also designed to scan objects with diameters up to $100 \mathrm{~mm}$, for other engineering applications. Several main factors were considered during the design stage that could have affected the performance of the rig:

- Overall stability of the rig: a lightweight base with poor stability may lead to significant errors in image capture and loss of calibration whenever the rig is moved or touched;

- Camera movement systems: non-smooth and non-stable movement of the camera will also result in the loss of camera calibration.

The complete flow chart and actual photo of the vision system are shown in Figs 11 and 2 respectively.

\subsection{The rig features}

\subsubsection{Structure}

The structure is able to support at least two cameras at any one time; for greatest accuracy, the structure is extremely stable so that there is no movement when the cameras are taking images; it is significant enough for the cameras to be able to take images of objects up to $100 \mathrm{~mm}$ in diameter. Based upon the design criteria set out, several design concepts were conceived. The concept, to set cameras in opposite planes as shown in Fig. 3. was proven to be the most reliable: the vertical camera movement criteria are satisfied using this design, allowing the camera to move in the $z$ and $x$ axis. The horizontal camera movement would be limited to movement in the $x$ and $y$ axis, leaving only the $z$ axis movement unresolved. This problem was solved by designing an adjustable camera mount. This concept benefits from using two sliding tracks, which in this case, are mounted in opposite planes. Figure 3 shows the CAD model and the actual picture of the rig structure.

\subsubsection{Camera movement}

The cameras are able to move towards and away from the object in both the vertical and the horizontal planes; the cameras are able to move to any distance from the object within the constraints of the rig size, which improves the accuracy and aids the automation process. For the camera movement 


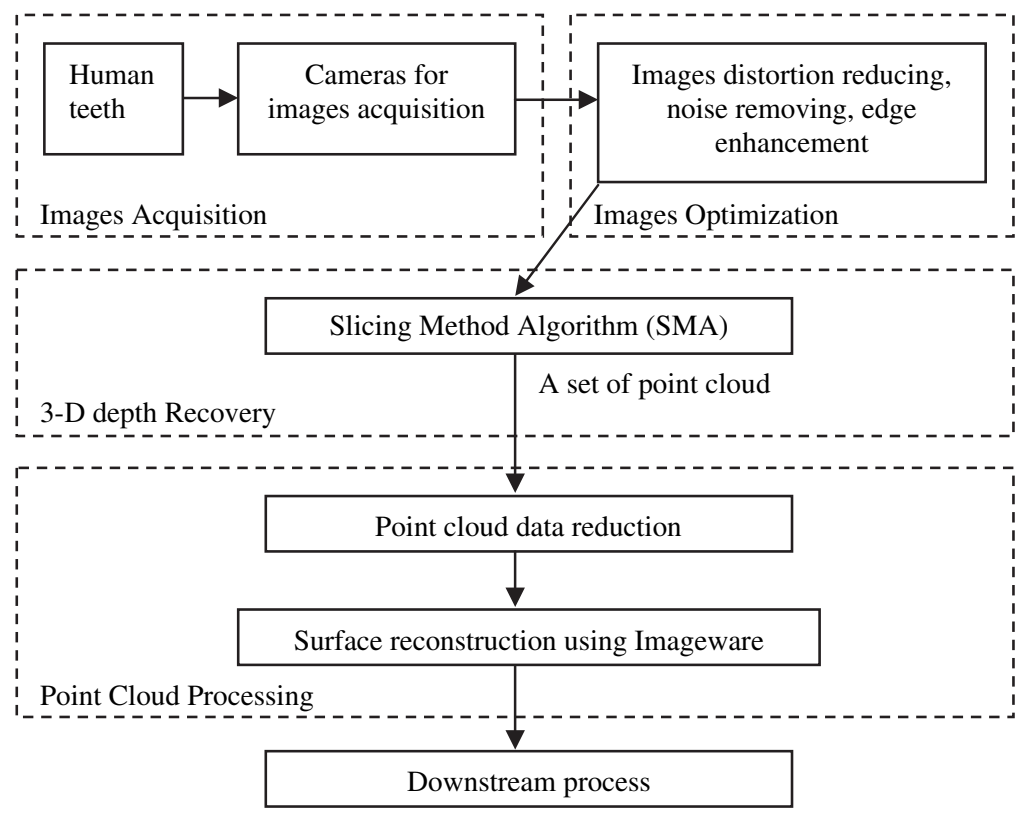

Figure 1: The flow chart of the vision system.

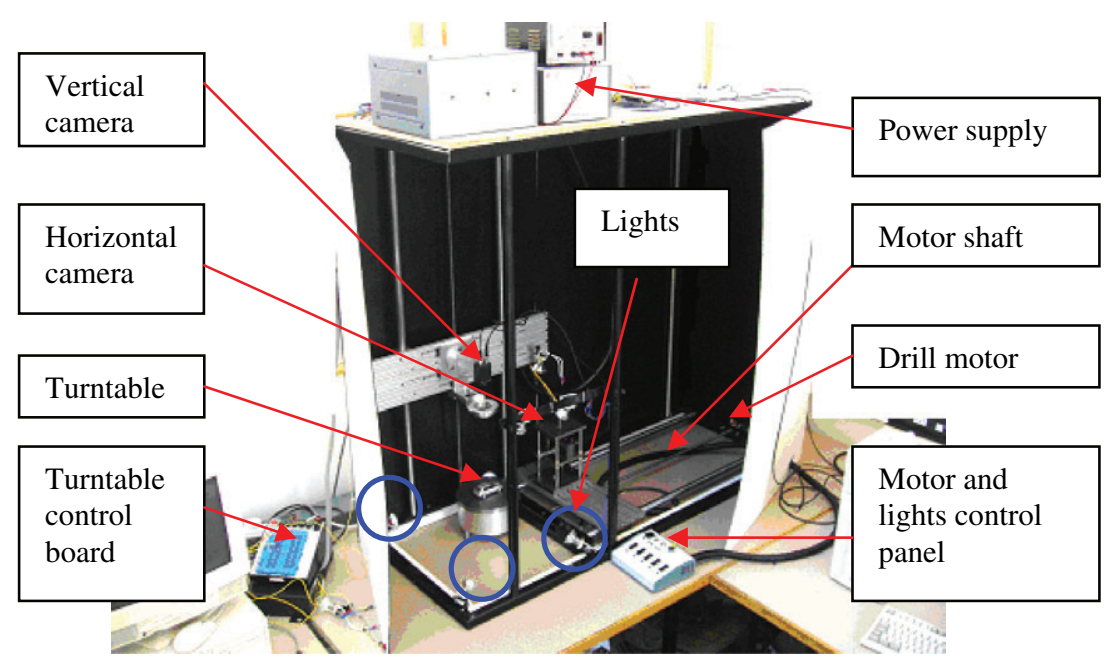

Figure 2: The actual photo of the vision system.

systems, many options were considered. It was decided to use two cylindrical runner bars with sliders running along them.

Figure 4h shows sliders manufactured from a solid a block, in which a hole along the centreline was milled, toleranced to a running fit. Using a solid rectangular block also allows them to be mounted to the back of the prefabricated aluminium track, usually called ITEM track, which is commonly used 

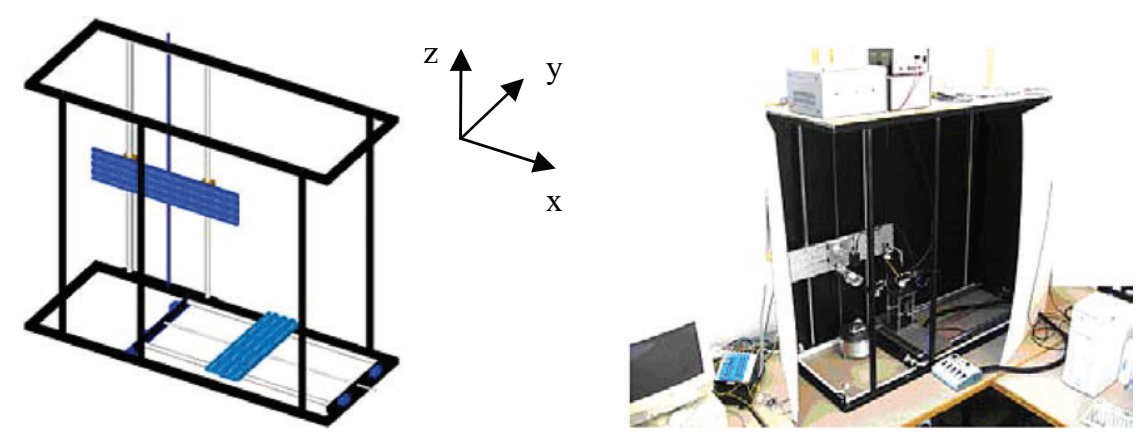

Figure 3: The CAD model and the actual picture of the rig structure.

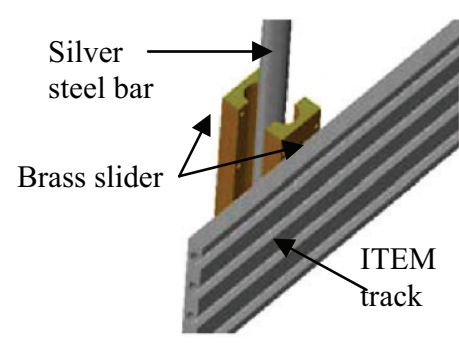

(a)

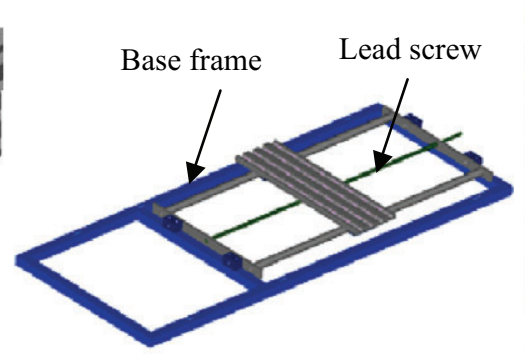

(b)

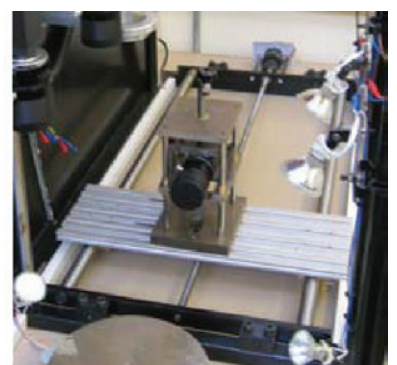

(c)

Figure 4: (a) Slider manufactured from solid brass block, (b) finalized design solution of the base frame, and (c) the horizontal camera system and the drill motor.

for building machine systems and test rigs. Brass was used to make the sliders and silver steel for the runners, allowing a smooth sliding mechanism.

The camera movement system was developed further to allow the end of the runners to be moved parallel to each other. This was required to ensure that the system would slide smoothly, should there be any error in the accuracy of the manufacture of the main structure. To allow this adjustment, the runners had guide bars at each end, which could be moved in a direction perpendicular to that of the runners. The lead screw as shown in Fig. $4 \mathrm{~b}$ in the horizontal track is driven by a drill motor (Fig. 4c). One of the aims set out at the start of the design was to ensure that the prototype had a modular design, which allowed the camera movement systems to be removed from the main frame without either system being fully dismantled. Figure $4 \mathrm{c}$ shows the final horizontal camera movement system attached to the bottom framework. The vertical system uses a similar design but the only difference is the vertical system mounts two cameras whereas the horizontal system mounts one.

One of the main decisions in finalizing the design was the travel required by the cameras. This, as set out in the design statement, must be great enough to cater to an object of a maximum diameter of $100 \mathrm{~mm}$. Using the cameras/lenses manufacturing data sheet, the calculations seen in Fig. 5] were carried out, the result giving a required distance of at least $835 \mathrm{~mm}$ from the CCD camera to the object. The distance of travel required by the system is larger than this to cater to both the size of the lens $(92.9 \mathrm{~mm})$ and the camera mounts. To give sufficient room, this length was extended to $1,000 \mathrm{~mm}$, thus enabling the system to cater to objects ranging in size from less than $1 \mathrm{~mm}$ to $100 \mathrm{~mm}$ in diameter. 


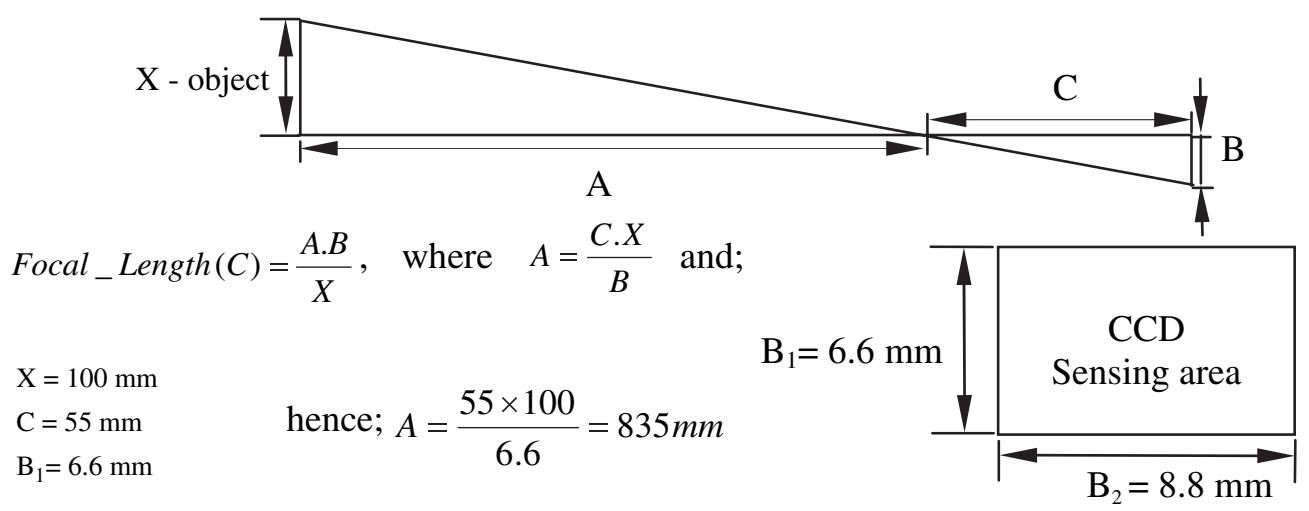

Figure 5: Calculation of camera travel distance.

\subsubsection{Camera mounting}

The main design objectives for both vertical and horizontal camera mounts are as follows:

- camera perpendicularity and stability - essential for accurate, calibrated images,

- accurate camera movement and rotation - particularly with the vertical camera mount to expand the system capability for the human based vision (i.e. stereo vision) method,

- fast camera movement and rotation,

- secure fixture of camera and lens - ensuring they will not be damaged, and

- minimal weight - particularly with the vertical camera mount.

\section{Vertical camera mount system}

Based on the design objectives listed above, the vertical camera mount system should have the ability to control the rotation of the camera (Fig. 6) similar to human vision, but more flexible. This was achieved by having two separate plates on one axle (a single bolt running through from the back face), where the outside plate (the disc to which the camera is attached) is free to rotate and can be locked in place by grub screws.

Engineering design methods [11] were used to establish a concept selection matrix in conjunction with product design specification (PDS) requirements to make a suitable choice and judgement on vertical camera conceptual designs of sub-systems. The t-slot turntable mount was chosen as it incorporated good reliability and safety characteristics with a smooth, precise rotating mechanism.

\section{Horizontal camera mount system}

Due to the many common design objectives, the designs for the vertical camera mount's base can also be considered for the horizontal camera mount. The only difference is the addition of a mechanism to adjust the camera's height relative to the object in accordance with the slicing method. The solution was to attach the camera to a platform that ran up and down guide bars (Fig. 7 ) to ensure that the camera is always aligned with the turntable centre. A threaded bar that attaches to the platform through a bearing could then be used to apply the necessary force to drive it up and down.

The free slider design was chosen as it allows support of the lens, which is more important than on the vertical camera as a larger moment is introduced (the largest lens weighs $0.942 \mathrm{~kg}$ and ordinarily 


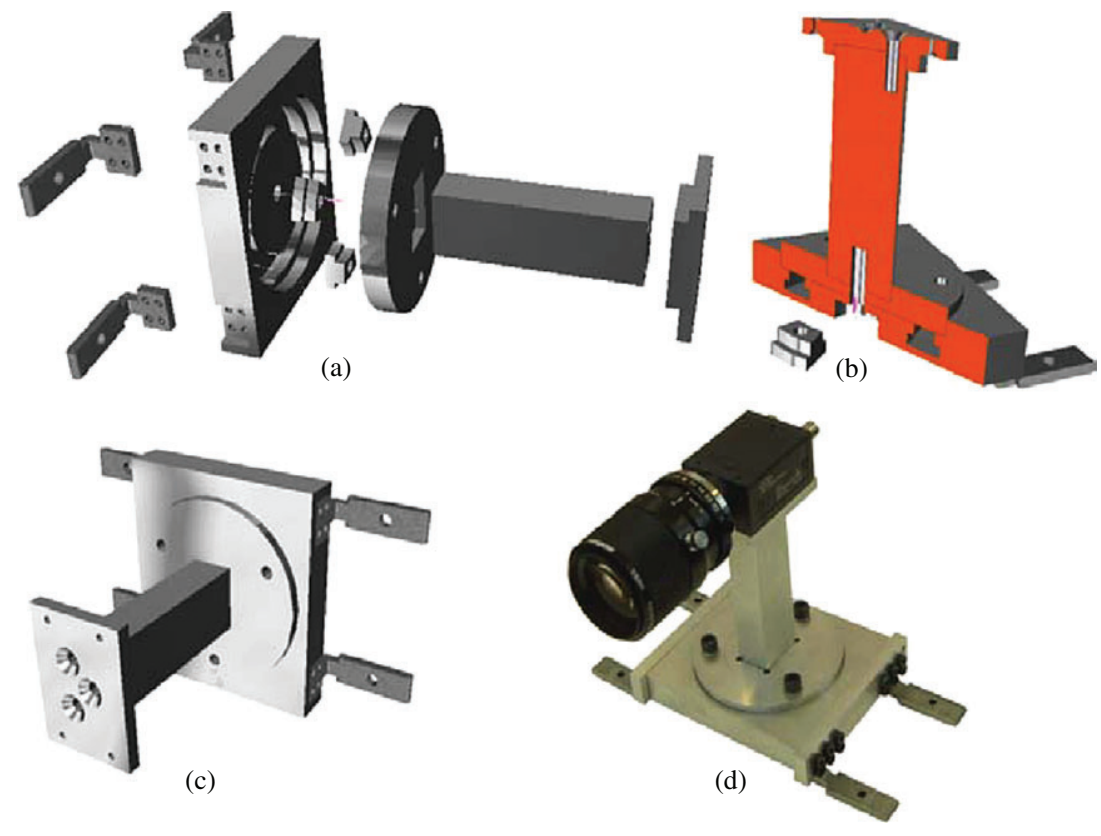

Figure 6: Vertical camera mount system: (a) exploded assembly view, (b) cross-section showing shaped groove, (c) assembly view, and (d) manufactured assembly.
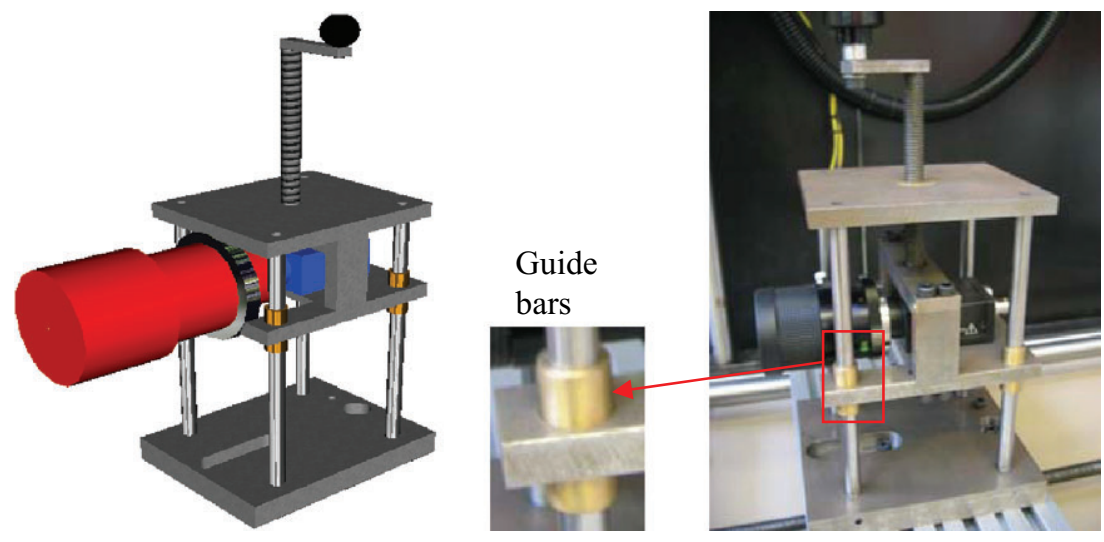

Figure 7: Horizontal camera mount system.

is only supported by the thread that screws into the camera). These supporting arms on the platform also aid lens alignment.

2.2.4 Turntable design for object rotation

The CAD model and manufactured turntable (the top plate was removed in order to have a clear image of the control mechanism) is shown in Fig. 8, 


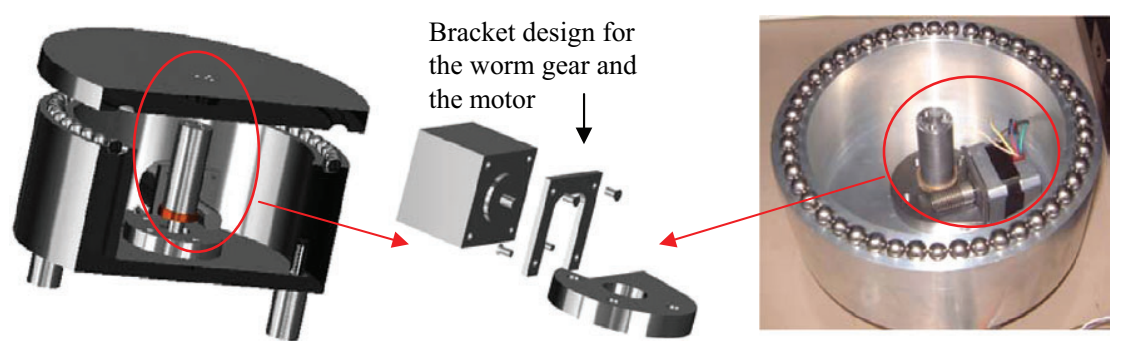

Figure 8: The CAD model and the manufactured turntable.

The following design objectives for the turntable holding the object were identified:

- Automated method of rotation. This includes:

- The ability to rotate in small increments $\left(\sim 4.5^{\circ}\right)$ through $180^{\circ}$.

- High level of stability. There should be no pitching of the disc as it rotates. The design must also ensure that the position of the turntable or the rig is not influenced in any way by the operation of the rig.

- Rapid rotation between shots. The amount of time for the object to be set up and rotated should be as low as possible.

- The revolving turntable disc must be large enough to accommodate an object being viewed from the maximum range of the camera. This will allow flexibility for scanning different objects in the future.

- The disc must incorporate a method of attaching a stand.

- Reliability. By keeping the design simple, the risk of failure will be minimized. In addition, the design must be able to withstand misuse.

- Manufacturing complexity should be minimized.

\subsubsection{Lighting}

Lighting is a very important factor in obtaining the correct type of image for each vision system technique. The lighting should be versatile and the rig must be able to accommodate as many different types and positions of lighting as possible to allow any technique to be used or object to be scanned.

The rig not only completely controls the light (by means of an enclosed structure) but is also flexible enough to hold several different types of light at various positions to optimize the image of the object (Fig. Q). For the slicing method, the ideal image is a silhouette of the object with sharp edges and no shadow, but for the human/stereo vision method, it is almost the exact opposite; shadow is essential to detect features that allow the computer to match the disparity between each image.

\section{CAMERA MOVEMENT AUTOMATION AND ITS CONTROL UNIT}

Automation of the camera movement was essential to reduce set-up time and increase accuracy. The motor selection for speed, maximum torque, and cost played an important role in designing the camera movement system. There were several factors to consider when choosing the motor: speed, maximum torque, and cost. Various motors and their technical specifications are listed in Table 1 The speed calculation showed that for the track to move from top to bottom in under $60 \mathrm{~s}$, a speed of around $800 \mathrm{rpm}$ was appropriate. The cameras should never actually need to move the whole distance at one time but it is useful to have that speed; for this reason, the Nylon geared motor was eliminated because it was too slow. The cameras, tracks, and sliders are heavy and the motor requires sizable 


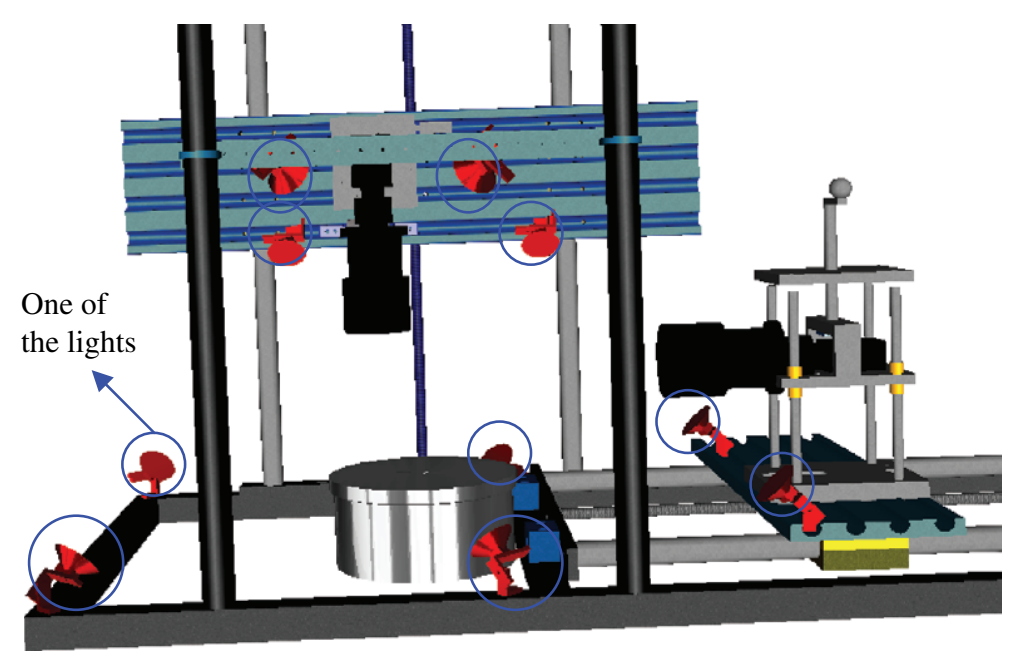

Figure 9: Lighting positions shown in the circles.

Table 1: Choice of motors and their technical specifications.

\begin{tabular}{lcccr}
\hline Type of motor & Voltage $(\mathrm{V})$ & $\begin{array}{c}\text { Maximum } \\
\text { speed }(\mathrm{rpm})\end{array}$ & $\begin{array}{c}\text { Maximum } \\
\text { torque }(\mathrm{mNm})\end{array}$ & Cost (£) \\
\hline Nylon geared light duty & 12 & 330 & 25 & 21.65 \\
22 mm Brush DC motor & 12 & 558 & 6.34 & 27.64 \\
RE 40 mm DC motor & 12 & 8,200 & 98.7 & 183.05 \\
Drill motor & 14.4 & 700 & 7,500 & 19.98 \\
\hline
\end{tabular}

torque to be able to move the system up and down. Calculation showed that a maximum torque of $6.46 \mathrm{mNm}$ is required. This eliminates the $22 \mathrm{~mm}$ brush DC motor because it only has a maximum torque of $6.34 \mathrm{mNm}$, which is not sufficient to drive the system.

The RE $40 \mathrm{~mm}$ DC motor is an ideal choice as it has a very high speed and large maximum torque. Unfortunately, it is expensive and two motors are required. For these reasons, we selected a common drill motor; it came with an added bonus of having a keyless chuck to attach the lead screw shaft directly to it, making the problem of mounting the motor fairly simple.

The motor mounts were designed and manufactured from an aluminium plate connected directly to the motor; the plate was connected either to the guide bar (for the horizontal mount) or directly to the frame (vertical mount) with two threads covered with a brass tubing to aid stability. The finished design can be seen in Fig. 10 .

A manual control was designed using three switches and a motor speed control. A relatively simple set-up was designed with a main power switch, a switch to choose which motor to drive (this was a safety feature so that both motors could not run at the same time), a switch to decide in which direction to drive each motor, light emitting diodes (LEDs) to indicate to the user which motor is on and which direction it will move in, and, finally, a motor speed control, which is essential to obtain the sharpest image. This was basically a voltage regulator with a potentiometer in series. There were also five light switches to control various combinations of lighting. The control unit can be seen in Fig. $10 \mathrm{~b}$. 
(a)

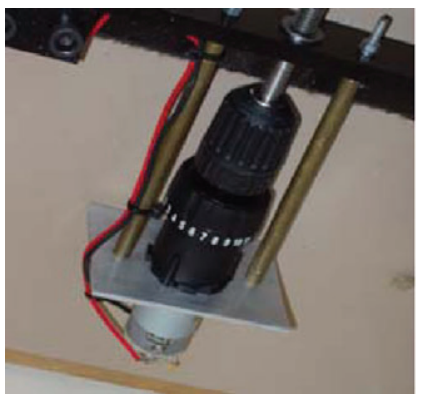

(b)

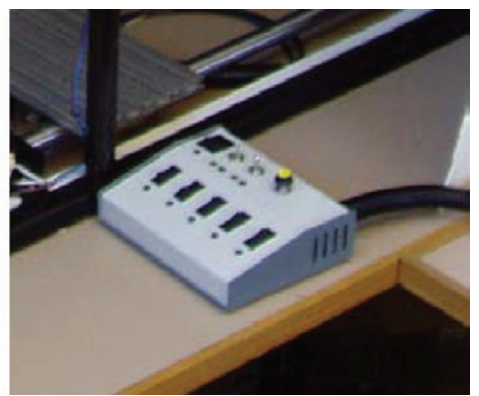

Figure 10: Photographs of (a) the motor mount and (b) the motors and the lighting control unit.

\section{IMAGE-BASED 3D DEPTH RECOVERY METHOD}

The image-based 3D depth recovery method, also known as shape-from-X technique, exploits a large variety of image cues related to various optical methods in vision system techniques. The optical methods can be described as active or passive processes, considering whether or not the method requires modification of the vision system's parameters [12] and lighting. The occluding boundary technique and its derived method, the slicing method, were the two 3D depth recovery methods used in this study. These two active methods usually consist of one camera, a turntable, and lighting [9]. The 3D depth recovery process consists of two major steps: (1) image capture and optimization and (2) depth recovery as shown in Fig. 1.

\subsection{Camera calibration}

The aim of camera calibration is (1) to reduce/eliminate the lens distortion and non-linearity of the CCD camera, which would influence the accuracy of the image [13, 14]; (2) to determine the internal camera's geometric and optical characteristics (intrinsic parameters) and/or the 3D position (translation) and orientation (rotation) of the camera frame, relative to a real world coordinate system (extrinsic parameters). Various efficient camera calibration methods have been developed during the last decade [15, 16]. Bouguet's [15] Matlab camera calibration toolbox was used to compute the camera calibration matrix [17] to reduce the distortion of the profile images captured by the horizontal CCD camera.

\subsection{Point cloud extraction}

\subsubsection{Occluding boundary and slicing method}

In both the occluding boundary and the slicing methods, the object is rotated in different angles, a number of profile views/images can be taken and the objective is to recover the shape from these profile views. For each view, the silhouette (3D boundary) of the object is extracted using the edge detection technique [18]. The occluding boundary method can be applied to objects without regional concaved surfaces and to almost any solid material or biomaterial such as sunflower seed [19] (because only the silhouette of the object is to be detected), which can be recognized as one of the advantages of this optical method. The more complex the shape, the more angles are needed and this is determined by the accuracy required by the $3 \mathrm{D}$ model. These profile views can be used to create a series of occluding boundaries. Each occluding boundary is effectively the rim of the object projected in the image plane. The slicing method is currently considered as the most accurate and versatile one amongst those methods derived from the occluding boundary. Figure 11 shows the visual perception process 


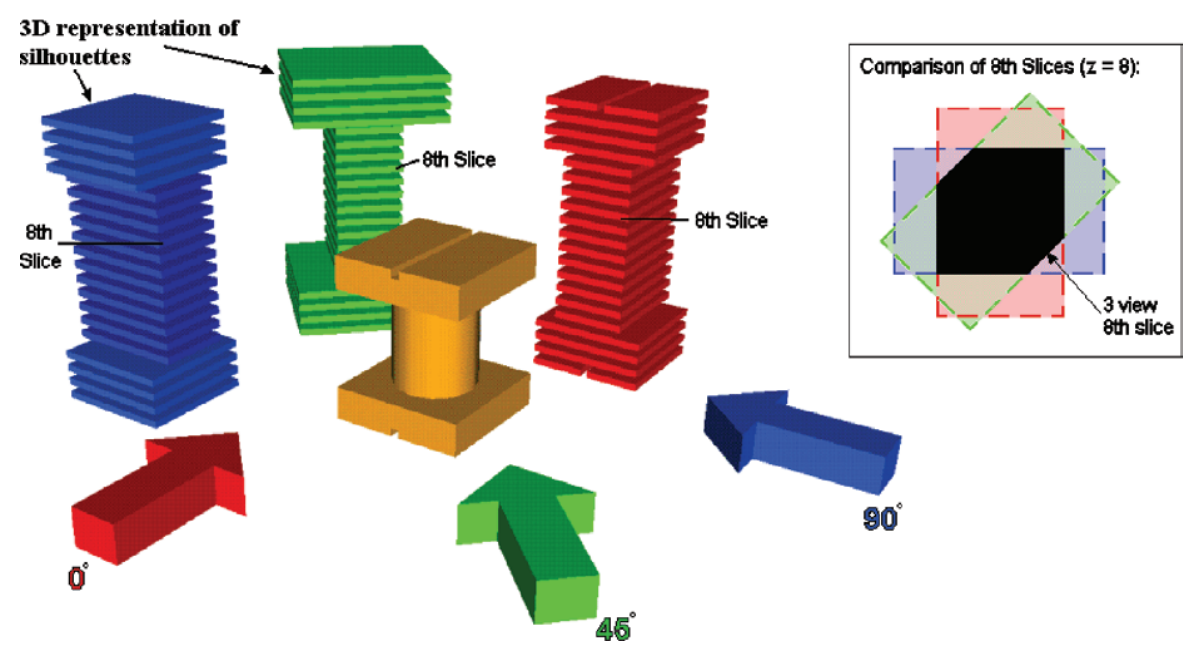

Figure 11: Visual representation of the slicing method: the boxed section shows an example union operation with the eighth slice.

of the slicing method. However, the slicing method is currently incapable of extracting information for concave surfaces. All these surfaces will be recognized as plain surfaces; therefore, the slicing method is good at processing teeth like incisors and canines but is not capable of processing molars with a concave surface occlusal. This problem can be overcome by introducing two/multiple view methods [17] such as human based vision (i.e. stereo vision) method with two CCD cameras, which is the subject of current development.

In the slicing method (Fig. 11), a number of profile views of an object are captured from different angles. As in all occluding boundary techniques, the images are then discretized (edge detected), so that each image of the object is a silhouette. Each silhouette is then 'sliced' into one pixel high segments, which can then be projected onto a new image. This new image is a cross-sectional slice at a specific height of a particular view. Once a complete set of slices exists for each view, a union operation can be performed on corresponding slices from all the views. When all union operations have been completed, a final set of slices will have been produced that contain the data for the 3D model of the object.

\subsubsection{The edge detection technique}

To obtain the silhouette of each profile view image, it is necessary to determine the edge points that represent the contours of the object. Let each image be a matrix $I[i, j]$. The edge points are found by an edge detector in Matlab using the 'Sobel' method function $\operatorname{EDGE}(I[i, j]$, sobel) [20]. Because the images taken from the CCD cameras are bitmaps (greyscale images), $I(i, j)$ is the value of the greyscale for each pixel in the image, ranging from 0 to 255 . The following steps are used to determine the edge points:

1. Generate the edge image $E[i, j]=\operatorname{EDGE}(I[i, j]$, sobel $)$, which is a binary image.

2. Filter this edge image $E[i, j]$ by the following detector

$$
E(i, j)= \begin{cases}1 & \text { if } E(i, j) \text { is an edge point } \\ 0 & \text { otherwise }\end{cases}
$$


The pixels in binary images are presented as black with value 0 and white with value 1 , where, in this study, black means background and white means the contours of the object. Figure 12a and $b$ shows the greyscale image of a seven-year-old child's cracked incisor and the edge points, respectively.

\subsubsection{The slicing method algorithm}

The slicing method algorithm (SMA) was developed using the Matlab image processing toolbox to reduce the numerical calculation time in the previous studies [21, 22]. Figure [13 shows the visual perception of the slicing method in processing a tooth preparation model. The following operations are used to extract the point cloud from the profile images in Matlab:

1. Read the profile images as a matrix $I_{k}[i, j], k \in(1, n)$, where $n$ is the number of profile images.

2. Determine the edge points using the general edge detector for each profile image to generate the edge points matrix $E_{k}[i, j]$.

3. For each $E_{k}[i, j]$, compute the most distance $D_{k}(i)$ among the edge points for each row, where $D_{k}(i)$ is the slice width at the height $i$ for each profile image as shown in Fig. 13c.

4. Generate a square slice image $S_{k, i}[m, m]$ as in Fig. $13 \mathrm{c}$ according to the slice width $D_{k}(i)$, where the pixels within the width are set to black with the value 0 and others are set to white with the value 1 , and rotate this image by $180 \times k / n$ degrees.
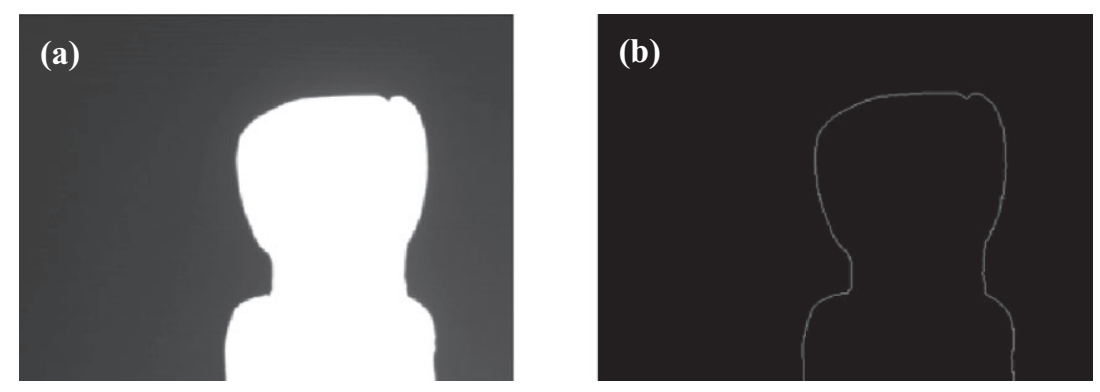

Figure 12: (a) The greyscale image and (b) the edge points.

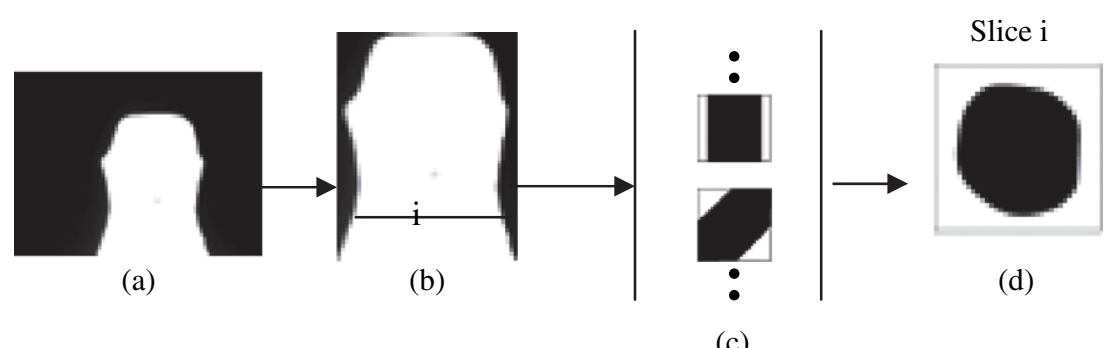

(c)

Figure 13: Slicing method in processing the tooth preparation model (example shown on a sample slice $i$ ): (a) profile images, (b) cropping and edge detection, (c) projection of each slice for every angle (slice $i$ ), and (d) intersection of every slice is the final slice of the tooth (slice $i$ ). 
5. For each $i$ (the same height at all the profile images), apply a union operator to the set of $S_{k, i}[m, m]$. The final slice $i$ can be written as

$$
\operatorname{SLICE}_{i}[m, m]=\sum_{k=1}^{n} S_{k, i}[m, m]= \begin{cases}1 & \text { if the point is within the slice } \\ 0 & \text { otherwise }\end{cases}
$$

6. Apply the edge detector to $\operatorname{SLICE}_{i}[m, m]$ to extract the surface points $P(x, y, z)$ of the object, where $z=i,(x, y)$ is the coordinate of the edge points of $\operatorname{SLICE}_{i}[m, m]$.

\section{VISION SYSTEM APPLICATION EXAMPLES}

A seven-year-old child's cracked incisor and a tooth preparation model provided by the first author and the Renishaw Triclone dental system [23] were digitized to evaluate the vision system using the SMA. These two samples were also scanned by the Renishaw Triclone dental system with $10 \mu \mathrm{m}$ accuracy (Fig. 14). The Triclone system's accuracy was that of compared with that of the vision system. This accuracy comparison was demonstrated as a disparity map with the vision system point cloud and the surface generated from the Triclone point cloud. (Fig. 15,

\subsection{Image acquisition}

Forty profile images were captured from each sample using a Bandit-II RGB frame grabber with a SONY XC-ST70 CCD camera and a Computar TEC M55 lens. The angle increment between each pair of neighbourhood images was $4.5^{\circ}$. The captured images had a resolution of $640 \times 480$. The CCD camera here had effective pixels of $768 \times 494$.

(a)

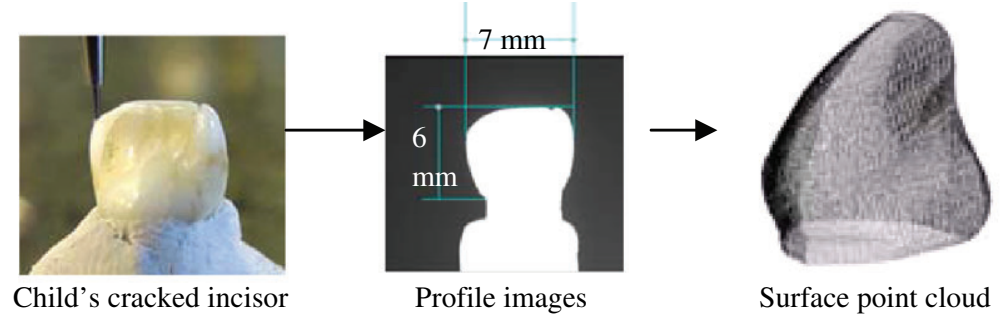

(b)

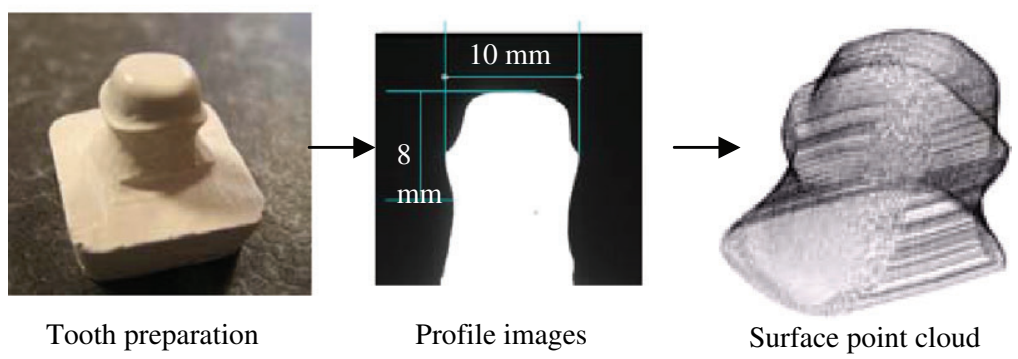

Figure 14: The results of the SMA on two samples. 
(a)

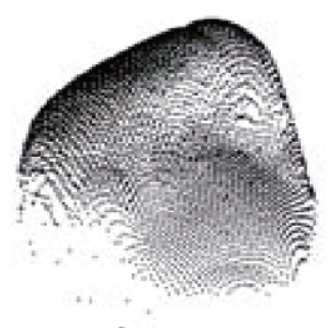

(b)

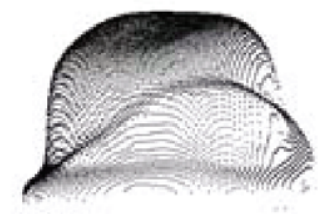

(c)

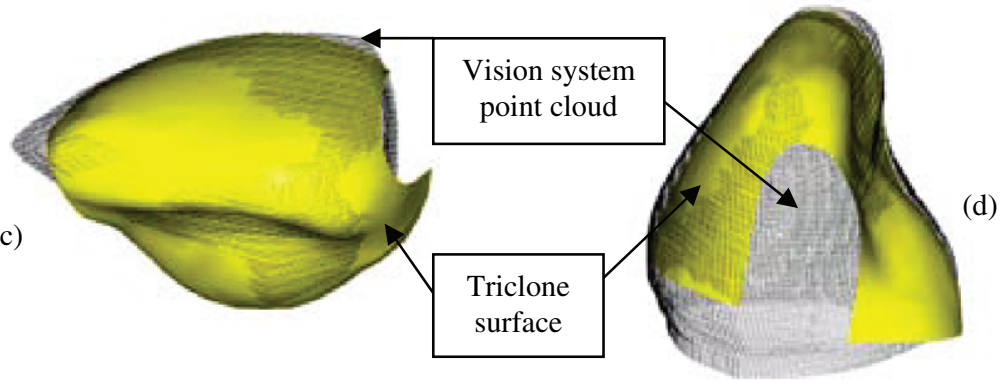

(e)

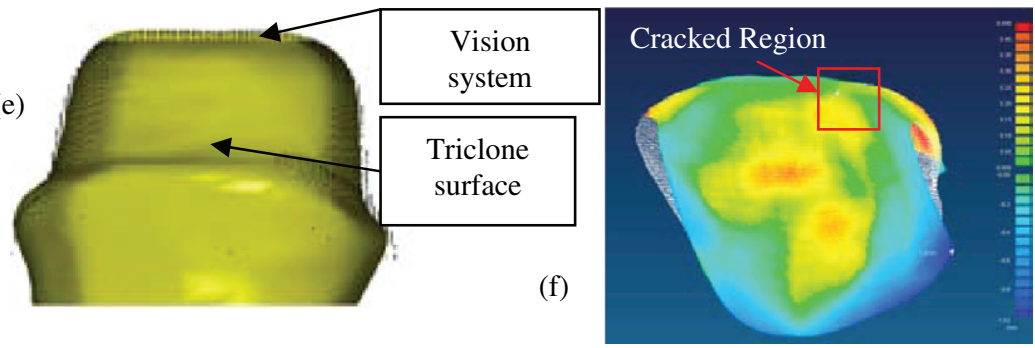

Figure 15: Evaluation of the SMA results: (a) child's cracked incisor point cloud: 6,103 points; (b) tooth preparation point cloud: 6,984 points; (c) comparison of the Triclone surface and the vision system point cloud (child's incisor, top view); (d) comparison of the Triclone surface and the vision system point cloud (child's incisor, profile view); (e) comparison of the Triclone surface and the vision system point cloud (tooth preparation, profile view); (f) disparity map between the Triclone surface and the vision system point cloud (incisor, average deviation: $147 \mu \mathrm{m})$.

\subsection{Image correction}

The calibration process described by Bouguet [15] was applied to reduce the distortion caused by the lens. The scale factors were then applied in both horizontal and vertical directions of real size dimension/pixel number. This was based on the scale factors calculated in both directions of profile images by carrying out a $2 \mathrm{D}$ dimension test, with each square test having the dimension of $5 \mathrm{~mm} \times 5 \mathrm{~mm}$.

\subsection{SMA results}

Figure $14 a$ and $b$ shows the process applied to the point clouds of the child's cracked incisor and the tooth preparation, respectively. The child's cracked incisor and tooth preparation were divided into 
349 and 487 slices, respectively, by the SMA. The cracked incisor point cloud had 208,631 points and the tooth preparation point cloud had 139,498 points. These point clouds had quite a large density and were filtered before surface reconstruction to reduce the noisy points (i.e. jagged edges), which were partly introduced by the low resolution of the images. This error can be overcome by higher resolution cameras and better edge enhancement. The cracked region of the child's tooth was the key area, which was given the greatest attention. This area's point cloud accuracy is discussed in Section 6. The SMA has the ability to interpret up to 1,000 slice images. On a Pentium P4 $2.6 \mathrm{GHz}$ platform and 1GB memory, the Matlab SMA took less than an hour in processing 500 slices, while it took around 2.5 hours in the previous project $[21,22]$ using the WIT (image processing software) environment. In a CMM or laser application, it may take several days to set up, calibrate the system, and scan. The vision system has shown its potential efficiency: the total processing time, from data capture to point cloud generation, was restricted to 2 hours and the scanning process was easier to manipulate.

\section{EVALUATION OF THE RESULTS}

These two samples were also scanned by the Renishaw Triclone dental system with $10 \mu \mathrm{m}$ accuracy; the results are shown in Fig. 15 The comparison result has been very promising for restorative dentistry. The vision system was proven to have an accuracy of within $150 \mu \mathrm{m}$ on the child's cracked tooth region and within $50 \mu \mathrm{m}$ on the tooth preparation model. The main sources of error are: (1) camera calibration error, mainly the scaling factors from image size (pixels) to real world dimension $(\mathrm{mm})$; (2) the uncertainty of the rotation axis $(<10 \mu \mathrm{m})$ of the object during the image acquisition process. There are several ways to reduce the errors:

1. Rotate the current scanning plane by $90^{\circ}$ to scan the tooth in its natural way to improve the accuracy on the cracked region.

2. Introduce higher resolution cameras; the current CCD camera only has effective pixels of $768 \times$ 494.

3. Increase the precision and accuracy of the turntable to acquire a more accurate view angle.

Comparing our vision system with a laser based optical 3D measurement dental system [24], the advantages are: (1) the point cloud generated by the SMA does not have scattered points; (2) the SMA is more suitable when processing a single tooth/coping and is capable of scanning a complex curved surface (except concave surfaces); (3) greater flexibility as laser light is not required; therefore, the working condition/environment of our vision system is safer and less limited.

\subsection{Dental vision based CAD/CAM}

\section{DOWNSTREAM PROCESS APPLICATIONS}

Figure 16 shows the tooth preparation point cloud optimized and surface reconstructed using Imageware [25]. The surface model was imported into the CAD/CAM system (i.e. Unigraphics) [26] to create the CAD model, which was then transferred to a computer numerical control $(\mathrm{CNC})$ machine. The top part of the tooth preparation was machined.

\subsection{Finite element analysis}

Knowledge of a finite element analysis on a 3D tooth model has significant importance to improve durability and patient care in restorative dentistry, where a precise tooth model is one of the most important pre-conditions to improve the biomechanical performance [5]. 


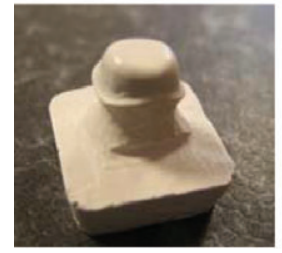

Tooth preparation

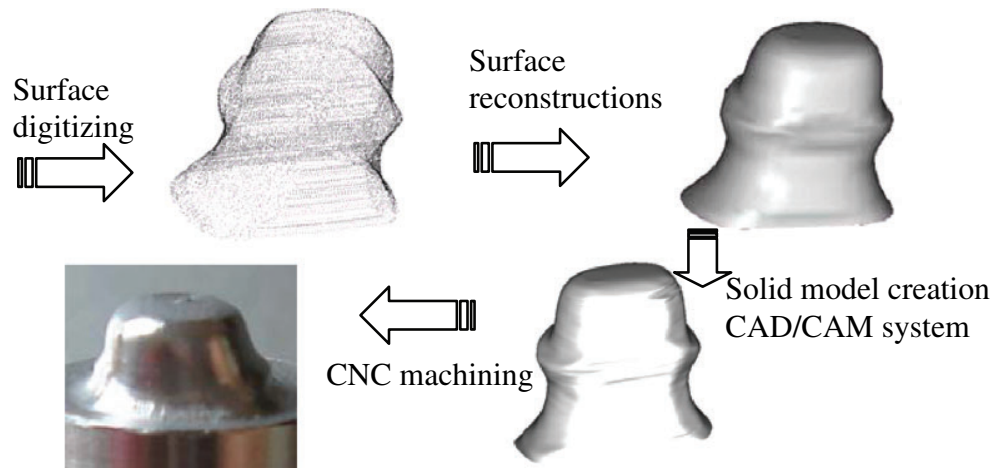

Figure 16: Process of dental vision based CAD/CAM system.

\section{CONCLUSION AND FUTURE WORK}

A vision rig prototype has been designed, fabricated, and tested on two complex objects (i.e. the child's cracked incisor and the tooth preparation) in dentistry. One of the advantages of the developed optical device is that it can be applied to bioengineering objects without regional concaved surfaces and almost any biomaterial such as sunflower seed [19]. The developed vision system can also be applied to insect wing motion simulation systems which has important industrial, civil, and military applications. The vision system will be used in an attempt to digitally capture the insect wing and hinge shape [27], which consists of a relatively complex biological structure that conventional CAD cannot model directly, and both CMM and laser scanners are incapable of digitizing. Current development concentrates on a final integrated system, incorporating the stereo vision technique based on the human vision principle and LED technology. It can be concluded that the vision system could encode the shape of complex bioengineering objects and the device could be useful in the characterization of natural materials as a basis for biology based design.

\section{ACKNOWLEDGEMENT}

We would like to thank Renishaw plc for donating the Cyclone scanning machine and various other dental materials and for their constant support throughout the project.

\section{REFERENCES}

[1] Armstrong, P.J. \& Antonis, J., The development of an active computer vision system for reverse engineering. Proceedings of the Institution of Mechanical Engineers, Part B: Journal of Engineering Manufacture, 214, pp. 615-618, 2000.

[2] Aoyama, H. \& Suzuki, Y., Autonomous measurement of physical model shape for reverse engineering. Journal of Manufacturing System, 19(6), pp. 375-381, 2000.

[3] Malamas, E.N. \& Petrakis, E.G.M., A survey on industrial vision systems, applications and tools. Image and Vision Computing, 21, pp. 171-188, 2003.

[4] Schmidseder, J., Aesthetic Dentistry: Color Atlas of Dental Medicine, Thieme: New York, 2000.

[5] Lin, C.L., Chang, C.H. \& Ko, C.C., Multifactorial analysis of an MOD restored child premolar using auto-mesh finite element approach. Journal of Oral Rehabilitation, 28, pp. 576-585, 2001.

[6] Paulus, D., Wolf, M., Meller, S. \& Niemann, H., Three-dimensional computer vision for tooth restoration. Medical Image Analysis, 3(1), pp. 1-19, 1999.

[7] Duret, F., CAD/CAM in dentistry. J. Amer. Dent. Assoc., 117, pp. 715-720, 1988. 
[8] Chen, L.C., Development of innovative 3-D dental measurement technique and manpower for tooth model restoration through university-industry collaboration programmes. International Conference on Engineering Education, 2003.

[9] Klette, R., Schlüns, K. \& Koschan, A., Computer Vision: 3-D Data From Images, Springer: Singapore, 1998.

[10] Alves, N.M.F. \& Bártolo, P.J.S., Advanced reverse design through a new biologically based system. Design and Nature, 6, pp. 421-430, 2004.

[11] Wright, I., Design Methods in Engineering and Product Design, McGraw-Hill Publishing Co.: London, 1998.

[12] Trucco, E. \& Verri, A., Introductory Techniques for 3-D Computer Vision, Prentice-Hall: New Jersey, 1998.

[13] Sonka, M., Hlavac, V. \& Boyle, R., Image Processing, Analysis and Machine Vision, Chapman and Hall: London, 1995.

[14] Vision Elements, The Machine Vision Handbook, Firstsight Vision, 2005/2006, http:// www.firstsightvision.co.uk

[15] Bouguet, J., Camera Calibration Toolbox for Matlab, 2004, http://www.vision.caltech.edu/ bouguetj/calib_doc/

[16] Zhang, Z.Y., Flexible camera calibration by viewing a plane from unknown orientations. International Conference on Computer Vision (ICCV'99), pp. 666-673, 1999.

[17] Harley, R. \& Zisserman, A., Multiple View Geometry in Computer Vision, Cambridge University Press: Cambridge, 2003.

[18] Forsyth, D. \& Ponce, J., Computer Vision - A Modern Approach, Prentice Hall: New Jersey, 2003.

[19] Hernández, L.F. \& Bellés, P.M., A 3-D finite element analysis of the sunflower (Helianthus annulus L.) fruit under impact: a useful approach for the understanding and improvement of its hullability. Design and Nature, 6, pp. 253-263, 2004.

[20] Canny, J., A computational approach to edge detection. IEEE Transactions on Pattern Analysis and Machine Intelligence, 8(6), pp. 679-698, 1986.

[21] Alemzadeh, K. \& Zhang, L., A vision based reverse engineering approach to surface digitizing in restorative dentistry. Medical Image Understanding and Analysis 2005: Proceedings of the 9th Annual Conference, pp. 211-214, 2005.

[22] Alemzadeh, K \& Zhang, L., A vision based reverse engineering approach to surface digitizing in restorative dentistry. Proceedings of Sixth International Conference on Modelling in Medicine and Biology, Vol. VI, pp. 511-521, 2005.

[23] The application of science to dentistry, http://www.renishaw.com/client/product/UKEnglish/ PGP-1236.shtml

[24] Eva-Maria, B., Heike, R., Jens, A. \& Ralph, G.L., Analysis of three-dimensional sinter shrinkage of copings made from alumina in an innovative direct shaping process. Computerized Medical Imaging and Graphics, 28, pp. 159-165, 2003.

[25] UGS, Imageware, Imageware Course Guide: Modelling I, MDA 190-11, 2006, http:// www.ugs.com/products/nx/imageware/

[26] UGS, I-deas NX, 2006, http://www.ugs.com/products/nx/

[27] Burgess, S.C., Alemzadeh, K. \& Zhang, L., The development of a miniature mechanism for producing insect wing motion. Design and Nature, 6, pp. 237-244, 2004. 